\title{
BMJ Open Evaluation of the General Practice Pharmacist (GPP) intervention to optimise prescribing in Irish primary care: a non-randomised pilot study
}

\author{
Karen Cardwell, ${ }^{1}$ Susan M Smith, ${ }^{1}$ Barbara Clyne, ${ }^{1,2}$ Laura McCullagh,, \\ Emma Wallace, ${ }^{1}$ Ciara Kirke, ${ }^{5}$ Tom Fahey (D) , ${ }^{1}$ Frank Moriarty (D) , ${ }^{1}$ on behalf of the \\ General Practice Pharmacist (GPP) Study Group
}

To cite: Cardwell K, Smith SM, Clyne B, et al. Evaluation of the General Practice Pharmacist (GPP) intervention to optimise prescribing in Irish primary care: a non-randomised pilot study. BMJ Open 2020;10:e035087. doi:10.1136/ bmjopen-2019-035087

- Prepublication history and additional material for this paper are available online. To view these files, please visit the journal online (http://dx.doi. org/10.1136/bmjopen-2019035087).

Received 17 October 2019 Revised 12 February 2020 Accepted 25 March 2020

Check for updates

(C) Author(s) (or their employer(s)) 2020. Re-use permitted under CC BY-NC. No commercial re-use. See rights and permissions. Published by BMJ.

For numbered affiliations see end of article.

Correspondence to

Dr Frank Moriarty;

frankmoriarty@rcsi.ie

\section{ABSTRACT}

Objective Limited evidence suggests integration of pharmacists into the general practice team could improve medicines management for patients, particularly those with multimorbidity and polypharmacy. This study aimed to develop and assess the feasibility of an intervention involving pharmacists, working within general practices, to optimise prescribing in Ireland.

Design Non-randomised pilot study.

Setting Primary care in Ireland.

Participants Four general practices, purposively sampled and recruited to reflect a range of practice sizes and demographic profiles.

Intervention A pharmacist joined the practice team for 6 months (10 hours/week) and undertook medication reviews (face to face or chart based) for adult patients, provided prescribing advice, supported clinical audits and facilitated practice-based education.

Outcome measures Anonymised practice-level medication (eg, medication changes) and cost data were collected. Patient-reported outcome measure (PROM) data were collected on a subset of older adults (aged $\geq 65$ years) with polypharmacy using patient questionnaires, before and 6 weeks after medication review by the pharmacist.

Results Across four practices, 786 patients were identified as having 1521 prescribing issues by the pharmacists. Issues relating to deprescribing medications were addressed most often by the prescriber ( $59.8 \%)$, compared with cost-related issues (5.8\%). Medication changes made during the study equated to approximately $€ 57000$ in cost savings assuming they persisted for 12 months. Ninety-six patients aged $\geq 65$ years with polypharmacy were recruited from the four practices for PROM data collection and 64 $(66.7 \%)$ were followed up. There were no changes in patients' treatment burden or attitudes to deprescribing following medication review, and there were conflicting changes in patients' self-reported quality of life.

Conclusions This non-randomised pilot study demonstrated that an intervention involving pharmacists, working within general practices is feasible to implement and has potential to improve prescribing quality. This study provides rationale to conduct a randomised controlled trial to evaluate the clinical and cost-effectiveness of this intervention.
Strengths and limitations of this study

- This is the first study examining the role of general practice-based pharmacists in Ireland and the feasibility of evaluating this role.

- Integration of pharmacists was limited to four general practices, although these were diverse in terms of practice characteristics.

- A range of medication and patient-reported outcome measures data were collected, although because this was a pilot study, there was no control group to compare these with.

\section{INTRODUCTION}

The global burden of chronic disease is increasing, with more individuals living with long-term chronic conditions. Managing patients with multiple conditions (multimorbidity) and polypharmacy is recognised as a major challenge for healthcare systems. ${ }^{1}$ Within general practice, challenges include the fragmentation of healthcare across the primary-secondary care interface, singledisease clinical guidelines that do not reflect multimorbid patients, challenges in delivering patient-centred care and shared decision making. ${ }^{2}$ General practitioners (GPs) may also be coordinating prescribing from multiple specialists and attempting to balance benefits and risks from several medications. Thus, there has been an increased emphasis on the need to support GPs in the management of these patients. Appropriate medication use is one area of particular importance, ${ }^{3}$ and polypharmacy has been identified as a major priority by the WHO through its third global patient safety challenge, Medication Without Harm. ${ }^{45}$

Interventions to improve appropriate prescribing include those aimed at GPs (eg, computerised decision support) ${ }^{6} 7$ patient 
educational interventions and changes to care delivery arrangements, such as staffing models or skills mix. ${ }^{8}$ Pharmacists, integrated within general practice as a member of the primary care team, may support appropriate medicines use and provide benefits to patients. ${ }^{9} 10$ General practice-based pharmacists exist in a number of health systems internationally, including Canada, the UK and the USA. Evidence from published evaluations suggests pharmacists in general practice can have a positive impact on clinical outcomes, such as blood pressure and glycosylated haemoglobin, ${ }^{11}$ and may reduce medicationrelated hospitalisations. ${ }^{12}$ They may also release GP capacity by reducing prescribing activities. ${ }^{13}$ The model of practice-based pharmacists may provide advantages over a community pharmacy service, including (co-)location, access to medical records to inform the quality and appropriateness of recommendations, the potential for formal and informal communication and discussion of the pharmacist's recommendations, and reduced fragmentation of care. ${ }^{1014-16}$

Unlike countries such as the UK, pharmacists in Ireland have not been formally integrated into the general practice team, nor do they have prescribing rights. This feasibility study is part of a programme of work that follows the Medical Research Council (MRC) guidelines on developing and evaluating a complex intervention. The ultimate aim is to conduct a definitive randomised controlled trial (RCT) to compare the effectiveness and costeffectiveness of a General Practice Pharmacist compared with usual GP care. Therefore, this study aims to assess the feasibility and potential cost and clinical effectiveness of pharmacists, working with GPs, to optimise prescribing in Irish primary care. ${ }^{17}$

\section{METHODS}

\section{Study design and setting}

This non-randomised pilot study was conducted following the principles of the Consolidated Standards of Reporting Trials guidelines extension for the reporting of randomised pilot and feasibility studies. ${ }^{18}$ Ethical approval was granted by the Irish College of General Practitioners, and the study protocol has been published previously.

This study was conducted in general practice in Ireland, which has a mixed private and public healthcare system. The state provides some individuals with access to medical services, including hospital inpatient and outpatient care, GPs and dental services, free at the point of use through the General Medical Services (GMS) scheme. Approximately $40 \%$ of the population is eligible based on household income, with a higher threshold applying to those aged $\geq 70$ years so a greater proportion of this age group are covered. For people with income levels just above the GMS scheme threshold, a Doctor Visit Card (DVC) is granted, which entitles them to only GP visits free of charge. The Health Service Executive (HSE) is the main provider of health services, particularly in secondary care, while in primary care, GPs and community pharmacists are private contractors who provide services for the HSE. The remainder of the population not covered by state schemes pay out-of-pocket for their care or may have voluntary private health insurance that can cover some or all costs.

\section{Participants}

The study was conducted in four general practices. Practices were purposively selected from the national Primary Care Clinical Trials Network Ireland, to reflect a range of practice sizes and types from both socioeconomically deprived and affluent areas. Practices were invited to participate by email (which included a study information sheet, practice consent form and practice profile questionnaire) and a follow-up telephone call from the principal investigator (SMS). Consenting practices were visited by the study manager (KC) to discuss the logistics of the study and answer any further questions relating to their involvement in the study. Practice-related costs of participation in the study (room rental and GP time) were covered.

\section{Intervention components}

Following the enrolment of practices in the study, a pharmacist joined the general practice team for a period of 6 months working 10 hours per week, between September 2017 and March 2018. Pharmacists were recruited by the research team through an open recruitment process. The configuration of this time and activities in the practice were agreed between each practice and pharmacist. Unlike other countries, there are no formal training pathways or programmes for practice-based pharmacists in Ireland. However, each pharmacist was provided with a Study Intervention Manual, which detailed the scope of activities to be delivered by the pharmacist, based on national guidance and previous research. ${ }^{19-21}$ Broadly, this involved medication reviews (both opportunistic and targeted, conducted face to face with patients or using patients' medical charts only), involvement in the repeat prescribing process, conducting educational sessions with general practice staff and supporting GPs in undertaking clinical audits.

It was recommended that the medication reviews focused on three domains: (1) high-risk prescribing practices $^{22}$ and potentially inappropriate prescribing (PIP), ${ }^{23}{ }^{24}$ (2) deprescribing of medications that may cause harm or are no longer providing benefit ${ }^{25}$ and (3) rational and cost-effective prescribing, including use of 'preferred drugs' in accordance with recommendations from the HSE Medicine Management Programme (MMP). ${ }^{19}{ }^{20}$ The Preferred Drug Initiative has identified a single 'preferred drug' within several therapeutic drug classes, based on clinical and cost-effectiveness, which prescribers are recommended to use where possible, for example, lansoprazole as the preferred proton pump inhibitor. The MMP also provides recommendations on which dose-equivalent inhalers for obstructive airway conditions are most cost-effective. Pharmacists used 
indicators prespecified in the Study Intervention Manual to screen the medical records of the practice patient population and identify potential issues. Pharmacists do not have prescribing rights in Ireland, and therefore, the GP maintained clinical autonomy and implemented any changes to prescriptions, typically with non-urgent issues addressed at patients' next appointments and patients being contacted where more immediate changes were required.

\section{Outcome data collection \\ Prescribing data}

Throughout the 6-month study intervention period, pharmacists collected demographic and medication data for various prescribing indicators (as defined in the Study Intervention Manual) relating to adults patients of any age. These prescribing indicators were related to: (1) prescribing practices that were considered potentially inappropriate (ie, PIP) or high risk, (2) instances where medicines could be appropriately deprescribed and (3) instances where preferred drugs could be prescribed instead of non-preferred drugs. These data were collected while undertaking chart-based and face-to-face medication reviews, were anonymised with no patient identifiers and were recorded in a predefined data collection sheet. Online supplementary eTables 1-3 describe the prescribing indicators used in this study.

\section{Patient-reported outcome measure (PROM) data}

PROM data were collected in addition to the prescribing data from medication reviews to assess the feasibility of such data collection in a future randomised trial. In month 4 of the study period, eligible patients were invited to have a medication review conducted by the pharmacist and were asked to complete a patient questionnaire before and 3 months after this review.

Using the practices' prescribing software, pharmacists compiled a list of patients aged $\geq 65$ years who were taking $\geq 10$ repeat medicines. Thereafter, these patients were screened by a GP to determine whether they met any exclusion criteria and would be able to provide informed consent and participate in data collection. Patients were excluded if they had psychiatric or psychological morbidity or cognitive impairment sufficient to impair the provision of informed consent, a terminal illness likely to lead to death or major disability during the study follow-up period, or if they had already been reviewed by the pharmacist. Eligible patients were invited to participate via a letter (sent from the general practice) and received a follow-up call from a member of the practice administration staff. Following the provision of informed consent, patients were scheduled for a face-to-face medication review with the pharmacist. Chart-based medication reviews were completed for those patients who declined to attend for a face-to-face medication review. Chart-based reviews and lowering the repeat medicines threshold to $\geq 7$, and then $\geq 5$ medications, were protocol amendments implemented due to poor response rates.
The baseline patient questionnaire included questions on demographics, healthcare utilisation and PROMs relating to quality of life (EuroQol health-related quality of life five-level questionnaire (EQ-5D-5L) and visual analogue scale (VAS)) and medications (the Multimorbidity Treatment Burden Questionnaire (MTBQ) and the revised Patient Attitudes Towards Deprescribing (rPATD $)) .{ }^{26-28}$ The MTBQ is a 13-item questionnaire that measures treatment burden (the effort of looking after one's health) in patients with multimorbidity in primary care, while the rPATD questionnaire contains 22 items to capture older adults' beliefs and attitudes towards deprescribing. Only the PROMs were included in the follow-up questionnaire at 6 weeks postreview.

\section{Data analysis}

Descriptive statistics for general practices and patients with a prescribing issue were generated. For each prescribing indicator, we summarised the prevalence as a percentage of all the indicators within that category of prescribing issue. The proportion of cases where the implicated medication had been prescribed long term (ie, prescribed for $\geq 6$ months) and where the prescribing issue was addressed during the study period were also determined (ie, where a GP made some change to the medications following the pharmacist's intervention). Any changes occurring after the end of the 6-month study period were not captured. For PROM data collection, differences in outcomes reported prereview and postreview were summarised. For the EQ-5D-5L, participants were classed into those whose health state improved (improvement in at least one dimension and no worse in any other), worsened (worsening in at least one dimension and no better in any other), no change or mixed change (improved and worsened in different health states). ${ }^{29}$ MTBQ was summarised using median and IQR and by classifying into burden categories (none, low, medium and high), as recommended by the tool's developers due to nonnormality. ${ }^{27}$ Changes in EQ-VAS, EQ-5D-5L utility score (based on an Irish value set derived from a general population representative sample) ${ }^{30}$ and each dimension of the rPATD were examined using a paired-samples t-test. Data analysis was conducted using Stata V.14 and p values $<0.05$ were deemed significant.

A cost analysis was conducted to determine the costs saved or incurred for changes in prescribing due to the GPP intervention. This analysis was based on the cost of providing the intervention and the cost savings realised from the intervention. For each medication change detected during the study period, the total cost to the health system over 12 months was calculated. This cost included the publicly available drug reimbursement price (less wholesaler discount), ${ }^{31}$ as well as pharmacist dispensing fees of $€ 5$ per item (in line with the HSE dispensing fee structure). ${ }^{32}$ Where medications were amended, the cost difference between the original and new prescription was calculated. Costs of laboratory tests ordered as a result of pharmacist review were also 
determined. Where data on the original and/or new prescription were missing, the mean cost difference for changes within that indicator was used. This analysis only considered direct costs relating to medication changes and did not evaluate the downstream savings due to more appropriate prescribing, such as reduced medicationrelated hospitalisations.

\section{Continuation criteria}

Continuation criteria were used to determine whether further evaluation of this intervention is justified. These continuation criteria were outlined in the study protocol paper, ${ }^{17}$ based on consideration of the primary objectives around feasibility (including recruitment and retention of practices, pharmacists and patients) and the potential for effectiveness and system-wide implementation.

\section{Sample size}

Since this was a feasibility study, no formal power calculation was conducted. The recruitment target for the PROM data collection was 50 participants per practice (200 in total); however, this target was not reached.

\section{Patient involvement}

There was no formal public and patient involvement for this study; however, we engaged with the Collaborative Doctoral Awards in Multimorbidity PPI panel for consideration of the continuation criteria and input into the follow-on pilot cluster RCT.

\section{RESULTS}

Three pharmacists were integrated into four participating general practices for a period of 6 months; one pharmacist delivered the intervention in two practices. Pharmacists (one male, two female) had a mean of 15.7 years' clinical experience as a pharmacist prior to their involvement in the study. Two pharmacists worked in community pharmacy, with experience providing clinical services to local nursing home and community health settings. The remaining pharmacist was a prescriber (working in the UK) with experience in community and general practice settings, who worked primarily in academia. Recruited general practices were from both socioeconomically deprived and affluent areas (see table 1 for an overview of the characteristics of enrolled practices at baseline).

In three practices, pharmacists were based in their own room with their own computer and had access to patients' electronic medical records. In one practice, the pharmacist was based either in the practice administration office with the administration staff or in their own room, depending on the availability of practice rooms on that day. Tasks undertaken by the pharmacist varied across practices (which may have affected the results); however, in all cases, this included identifying potential prescribing issues (both those prespecified in the Study Intervention Manual and others based on their clinical judgement) and facilitation of practice audits. In addition, one

\begin{tabular}{|c|c|c|}
\hline $\begin{array}{l}\text { Characteristics of general } \\
\text { practices }(n=4)\end{array}$ & $\begin{array}{l}\text { Mean per } \\
\text { practice }\end{array}$ & Range \\
\hline Total number of GPs & 4.25 & $2-9$ \\
\hline Percentage of male GPs & 52.9 & $0-66.7$ \\
\hline Percentage full-time GPs & 88.2 & $77.8-100$ \\
\hline Number of GP sessions/week & 27 & $11-64$ \\
\hline Number of patients & 8830.5 & $1777-16631$ \\
\hline $\begin{array}{l}\text { Percentage of GMS-eligible } \\
\text { patients }\end{array}$ & 45.5 & $13.6-95.0$ \\
\hline $\begin{array}{l}\text { Percentage of patients aged } \\
\geq 65 \text { years }\end{array}$ & 16.1 & $10.8-24.8$ \\
\hline
\end{tabular}

GMS, General Medical Services; GP, general practitioner.

pharmacist delivered practice-based educational sessions on the treatment and management of patients with chronic obstructive pulmonary disease or type 2 diabetes mellitus, using an electronic prescribing tool developed by the pharmacist and a GP at that practice. Pharmacists' recommendations were made to GPs either face to face or via the internal email system on the practice prescribing software, depending on the nature and urgency of the recommendation. In the latter case, the colocation of pharmacists in the practice allowed for face-to-face discussion of recommendations when required. One intervention component (management of repeat prescribing) was not delivered by any pharmacist at any recruited practice, as this process had been standardised and was operating successfully within each practice.

\section{Medication reviews}

Pharmacists identified 786 patients with one or more prescribing issue during chart-based or face-to-face medication reviews, the majority via chart-based review $(\mathrm{n}=748)$. The mean age was 69.8 years $(\mathrm{SD} 14.8)$, and $65.2 \%$ were female $(n=513)$. The majority $(649,82.4 \%)$ were GMS patients, while $42(5.3 \%)$ were DVC patients. Patients were on a mean of 9.5 medications (SD 5.5), with a mean of 4.7 medications (SD 3.6) prescribed generically.

A total of 1521 potential issues were identified (table 2), a total of $59.6 \%$ relating to high-risk or potentially inappropriate prescribing, $9.5 \%$ where medicines could be deprescribed and $31.0 \%$ where a non-preferred drug was prescribed. The most common PIP/high-risk prescribing indicators identified involved long-term proton pump inhibitors at maximal dose, short-acting benzodiazepines, non-steroidal anti-inflammatories, the prescribing of duplicate therapeutic classes in the same patient and tricyclic antidepressants. Most medications involved had been prescribed for $>6$ months (online supplementary eTable 4). Among the most common prescribing indicators, duplicate therapeutic drug classes were most commonly addressed (within the study period) following the pharmacist's intervention (in $87.9 \%$ of cases), while 
Table 2 Prescribing issues identified by pharmacists during medication reviews (both chart based and face to face)

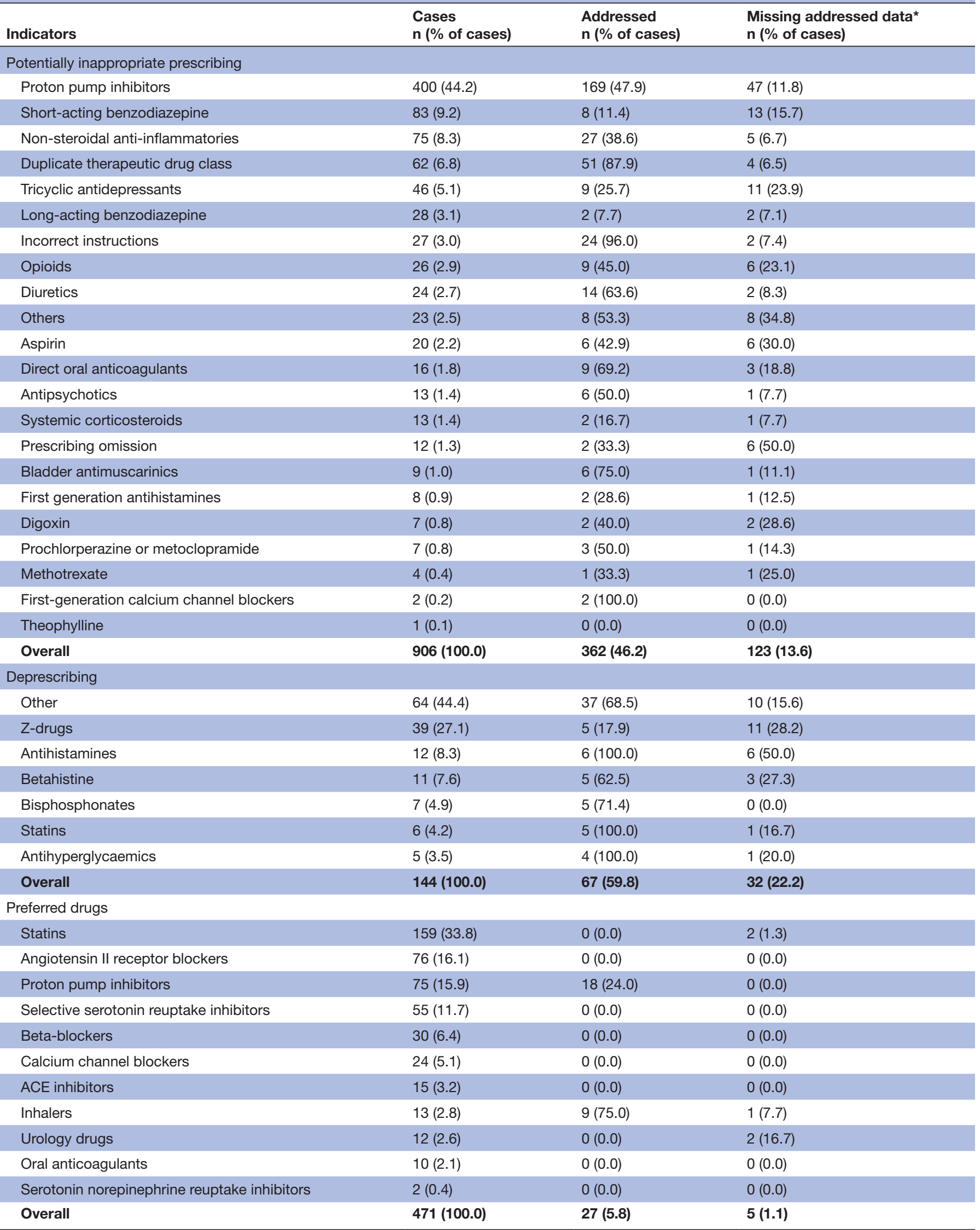

*Missing addressed data refers to cases where the status of whether a prescribing issue has been addressed was not recorded. 
short-acting benzodiazepines were least frequently addressed (in $11.4 \%$ of cases). The most common deprescribing indicators identified were 'Other' (this category included several drugs that were no longer required by/ indicated for the patient for example, quinine sulphate, cyclizine, domperidone, ferrous fumarate and valsartan), z-drugs, antihistamines, betahistine and bisphosphonates. All medications involved had been prescribed for $>6$ months. In relation to medications that could be deprescribed, other drugs (most often calcium and vitamin D combinations), z-drugs, antihistamines and betahistine were most commonly identified by the pharmacist. All of these were addressed by the prescriber through dose reduction or stopping in the majority of cases, with the exception of Z-drugs that were only addressed in $17.9 \%$ of cases. The most common drug categories in which nonpreferred drugs were prescribed were statins, angiotensin II receptor blockers, proton pump inhibitors, selective serotonin reuptake inhibitors and beta-blockers. Among all the indicators identified, only non-preferred inhalers and proton pump inhibitors were addressed (in $75.0 \%$ and $24.0 \%$ of cases, respectively).

\section{Patient-reported outcome measures}

Ninety-six patients of a prespecified target of $200(48 \%)$ were reviewed as part of PROM data collection. Table 3 describes the characteristics of these patients, and their detailed selfreported healthcare utilisation over the 12 months prior to the study is included in the supplementary material. Sixtyfour patients completed a questionnaire 6 weeks following their medication review. Table 4 compares patients' quality of life and attitudes towards medicines prereview and postreview, while the distribution across dimensions and levels of EQ-5D-5L is shown in online supplementary eTable 5. There was a statistically significant reduction of 0.06 in EQ-5D-5L score (95\% CI -0.11 to -0.002); however, when measured using the EQ-VAS, quality of life increased by a similar magnitude $(0.06,95 \%$ CI 0.02 to 0.10$)$. Overall, there was no significant change in Multimorbidity Treatment Burden Questionnaire score postreview compared with prereview, and the distribution of patients across levels of burden did not change. Regarding attitudes towards deprescribing, patients scored the appropriateness and involvement factors higher than the burden and concerns factors; however, neither these nor the two global items changed significantly following the intervention.

\section{Cost data}

The total cost of three pharmacists' salaries across four practices was $€ 31200$. This was based on $€ 30 /$ hour for 10 hours/week over 26 weeks. Practice-related costs for study participation included room rental $(€ 15 /$ hour for 10 hours/week) and GP time for meeting with the pharmacist (€70/hour for 1 hour/week), amounting to $€ 22880$. Table 5 reports the cost savings realised from addressed prescribing interventions. Overall cost savings that would accrue over a 12-month period following prescription changes amounted to $€ 56669$.
Table 3 Characteristics of patients enrolled in the PROM study and who completed patient questionnaires

\begin{tabular}{|c|c|}
\hline Characteristic & $\mathbf{N}(\%)$ \\
\hline Age, mean (SD) & $77.7(6.4)$ \\
\hline Female gender & $63(65.6)$ \\
\hline \multicolumn{2}{|l|}{ Marital status } \\
\hline Married or cohabiting & $48(50.0)$ \\
\hline Widowed, divorced or separated & $32(33.3)$ \\
\hline Single/never married & $17(17.7)$ \\
\hline Irish nationality & $90(93.8)$ \\
\hline \multicolumn{2}{|l|}{ Education } \\
\hline No schooling or primary only & $31(32.3)$ \\
\hline Secondary level & $32(33.3)$ \\
\hline Third level & $33(34.4)$ \\
\hline \multicolumn{2}{|l|}{ Employment } \\
\hline Retired & $71(74.0)$ \\
\hline Employed or homemaker & $21(21.9)$ \\
\hline Other & $4(4.2)$ \\
\hline Private health insurance & $46(47.9)$ \\
\hline GMS scheme & $65(67.7)$ \\
\hline Doctor Visit Card & $19(19.8)$ \\
\hline Distance GP, median (IQR) & $2(1-4)$ \\
\hline Any GP visit in previous 12 months & $95(99.0)$ \\
\hline Number of visits, median (IQR) & 5.5 (3.5 to 8$)$ \\
\hline Any A\&E visit in previous 12 months & $27(28.1)$ \\
\hline Number of visits, median (IQR) & 1 (1 to 2$)$ \\
\hline Any outpatient visit in previous 12 months & $52(54.2)$ \\
\hline Number of visits, median (IQR) & $2(1$ to 6$)$ \\
\hline Any inpatient stay in previous 12 months & $29(30.2)$ \\
\hline Number of stays, median (IQR) & 1 (1 to 3 ) \\
\hline Number of inpatient nights, median (IQR) & 3 (1 to 8$)$ \\
\hline
\end{tabular}

A\&E, accident and emergency department; GMS, General Medical Services; GP, general practitioner; PROM, patient-reported outcome measure.

\section{Continuation criteria}

All continuation criteria, with the exception of two, reached the threshold of 'Proceed with RCT', including those relating to practice and pharmacist recruitment and retention, and intervention acceptability, feasibility and potential for cost savings (see online supplementary eFigure 1). Those relating to patient recruitment and retention for PROM data collection were at the level of 'Do not proceed with RCT unless problems can be overcome'.

\section{DISCUSSION}

\section{Summary of findings and context in the literature}

To our knowledge, this feasibility study is the first to introduce and evaluate the feasibility of pharmacists in the general practice setting to support prescribing in Ireland. A large number of prescribing issues were identified by the pharmacists; however, the extent to 
Table 4 Comparison of patients' perceived level of health and attitudes towards their medicines premedication and postmedication review with the pharmacist

\begin{tabular}{|c|c|c|c|c|}
\hline Patient-reported outcome measure & $\mathbf{N}$ & Prereview & Postreview & Mean difference $(95 \% \mathrm{Cl})$ \\
\hline \multicolumn{5}{|l|}{ EQ-5D-5L } \\
\hline Utility score, mean & 62 & 0.728 & 0.673 & $-0.056(-0.111 \text { to }-0.002)^{*}$ \\
\hline No change & $8(12.5)$ & & & \\
\hline Improve & $19(29.7)$ & & & \\
\hline Mixed change & $14(21.9)$ & & & \\
\hline EQ-VAS, mean & 60 & 0.641 & 0.701 & $0.061(0.018 \text { to } 0.103)^{\star}$ \\
\hline \multicolumn{5}{|l|}{ MTBQ } \\
\hline Score, median (IQR) & 64 & $5.8(0-11.5)$ & $5.8(1.9-16.3)$ & \\
\hline Burden categories, n (\%) & 64 & & & \\
\hline Medium burden & & $11(17.2)$ & $11(17.2)$ & \\
\hline High burden & & $6(9.4)$ & $10(15.6)$ & \\
\hline \multicolumn{5}{|l|}{ rPATD, mean } \\
\hline Burden & 54 & 13.03 & 13.31 & $0.28(-0.71$ to 1.26$)$ \\
\hline Appropriateness & 52 & 17.13 & 17.23 & $0.10(-0.73$ to 0.92$)$ \\
\hline Concerns & 54 & 12.00 & 12.33 & $0.33(-0.95$ to 1.61$)$ \\
\hline Involvement & 60 & 21.33 & 21.6 & $0.27(-0.62$ to 1.15$)$ \\
\hline Satisfaction & 62 & 4.13 & 4.11 & $-0.02(-0.29$ to 0.25$)$ \\
\hline Willingness & 63 & 4.54 & 4.37 & $-0.17(-0.36$ to 0.01$)$ \\
\hline
\end{tabular}

${ }^{*}$ Paired t-test $<0.05$.

†Using an Irish value set derived from a general population representative sample.

EQ-5D-5L, EuroQol health-related quality of life five-level questionnaire; EQ-VAS, EuroQol-visual analogue scale; MTBQ, Multimorbidity

Treatment Burden Questionnaire; rPATD, Revised Patients' Attitudes Towards Deprescribing.

which these were addressed by the prescriber differed depending on the nature of the issues, ranging from $7.5 \%$ of those relating to cost-effectiveness (ie, use of nonpreferred drugs within a class) to $51.8 \%$ of those relating to potentially inappropriate or high-risk prescribing.

Table 5 Cost savings (over a 12-month period) from prescribing interventions

\begin{tabular}{|c|c|c|}
\hline Cost category & Cost saving & Cost incurrec \\
\hline Cost savings for amended drugs* & $€ 6374$ & \\
\hline Cost savings for stopped drugs & $€ 43681$ & \\
\hline $\begin{array}{l}\text { Cost savings from interventions } \\
\text { lacking full information }\end{array}$ & $€ 7162$ & \\
\hline $\begin{array}{l}\text { Total cost savings for drug } \\
\text { intervention }\end{array}$ & $€ 57217$ & \\
\hline Cost of tests ordered & & $€ 549$ \\
\hline $\begin{array}{l}\text { Overall savings from } \\
\text { intervention }\end{array}$ & $€ 56669$ & \\
\hline
\end{tabular}

*Cost of amended drugs was $€ 31394$ before intervention and $€ 25019$ after intervention.
The medication changes detected during the study period equated to approximately $€ 57000$ in cost savings assuming they persisted for 12 months. However, it has been argued that cost-effectiveness analysis of practicebased pharmacists should also consider saving relating to potentially improved prescribing, such as from avoided hospitalisations from adverse drug events or improved clinical outcomes. ${ }^{33}$ Although this feasibility study was not adequately powered to detect statistically significant outcome changes, these findings are encouraging and warrant further investigation to test the effectiveness of the intervention in future randomised studies.

Two recent systematic reviews found mixed evidence regarding the benefits of pharmacists in general practice to support prescribing, which may depend on heterogeneity in patient population included (ie, those with specific medical conditions or generally at risk of medication issues), outcomes assessed (ie, clinical, surrogate or patient-reported outcomes) and to what extent pharmacists were integrated into the general practice setting. ${ }^{10}{ }^{11}$ In the present study changes to address even minor prescribing issues required action from a GP as, 
unlike some other jurisdictions, pharmacists do not have prescribing rights. As highlighted in our study, the extent to which issues were addressed by prescribers differed, with issues of safety (ie, potentially inappropriate or highrisk prescribing) addressed more than issues of cost.

There were low levels of changes in cases of nonpreferred drugs (based on effectiveness, safety and costeffectiveness) within a drug class being prescribed in our study. Non-preferred inhalers were changed in more than two-thirds of cases; however, this category was somewhat different, as it represented a change of formulation rather than other categories that involved a change of chemical entity, and was also the subject of an audit in one practice. Reviews on barriers and facilitators of deprescribing may offer some explanations for the relatively low uptake of these medication changes. ${ }^{3455}$ Evidence that it is often only after a medication problem has occurred that deprescribing is considered may suggest that cost savings to the health system is an insufficient motivator to switch medications for a patient who is a prevalent user if no issue has arisen. ${ }^{34}$ Similarly, fear of negative consequences of a change (ie, potential adverse reactions to a new agent) is another potential barrier. ${ }^{35}$ By contrast, the safety concerns in cases of high-risk prescribing may have been sufficient to outweigh these fears resulting in higher uptake of these recommended changes.

There was a low response from patients to face-to-face review invitations with pharmacists as part of the PROM data collection with a recruitment rate of $48 \%$. While qualitative evidence from England indicates that pharmacist-led polypharmacy medication reviews are a positive experience for older individuals, ${ }^{36}$ the patients in this current study likely already had high treatment burden and frequent routine visits to healthcare professionals, given that they were on a high number of medications. ${ }^{37}$ They therefore may have been reluctant to attend an additional appointment for the purpose of pharmacist review as part of this study. In addition, the burden of data collection as part of this study and unfamiliarity with the role of pharmacists in general practice may have hampered participation. The continuation criteria indicated the problems of patient recruitment and retention need to be overcome in order to proceed with further evaluation of the intervention. This will be addressed by recruiting patients at study commencement before a pharmacist is integrated into each practice to ensure an adequate sample is achieved and to facilitate alignment of medication review with other routine visits.

There was a significant improvement in quality of life as measured by the EQ-VAS following the medication review and examining changes across EQ-5D-5L health states indicated no prevailing pattern of change following our intervention. This was inconsistent with the index score from applying an Irish value set to the EQ-5D-5L responses; however, the use of an external value set introduces an external source of variance and the values and weighting of different dimensions may not reflect those of participants in this study. ${ }^{29}$ The EQ-VAS captures health more broadly than the dimensions included in the EQ-5D-5L ${ }^{29}$ and has diverged from EQ-5D-5L index score in a previous trial of medication reviews in older adults. ${ }^{38}$

\section{Strengths and limitations}

To our knowledge, this is the first study examining the role of practice-based pharmacists in Ireland and the feasibility of evaluating this role. We used a range of valid indicators of high-risk and potentially inappropriate prescribing to guide pharmacists' interventions and to measure the potential clinical benefits. This pilot study was limited by its small sample size and predesign and postdesign in relation to patient-reported outcomes without inclusion of a control group; however, this was appropriate given the aim to assess feasibility. In addition, the generalisability of this study is limited by inclusion of only four purposively selected general practices. While uptake of pharmacist recommendations in relation to preferred drugs was low, a limitation of this study is that analysis only focused on prevalent users of relevant drug classes, and therefore, potential influence of pharmacists on prescribing in cases of new initiations could not be captured. The 6-month duration may mean that there was insufficient time to capture all prescribing changes made on foot of pharmacist recommendations during the study. The cost data captured was in the context of this research study and did not account for real-world employment costs that would be associated with the introduction of such a role. We also did not have any public or patient involvement in the study design and conduct; however, this is a key component of the planned randomised pilot study.

\section{Conclusions}

This study found that the integration of pharmacists, working with GPs, to optimise prescribing in Irish primary care is largely feasible and has potential clinical and cost benefits. A qualitative evaluation of this feasibility study is ongoing to explore this role further and inform future research. In line with the MRC guidelines on developing and evaluating complex interventions, this will now proceed to a randomised pilot study, with changes to the intervention and study design informed by the results of the present study (in particular, relating to patient recruitment) and qualitative evaluation. Patient recruitment ahead of integration of pharmacists will allow for earlier engagement between pharmacists and patients and the potential for enhanced follow-up after medication review. This will provide further evidence on the role of pharmacist in GP practices in the Irish context and the potential of this intervention to help achieve the ambitious target of the WHO's Medication Without Harm challenge to reduce serious, avoidable medication-related harm by $50 \%$ in 5 years. ${ }^{4}$

Author affiliations

${ }^{1}$ HRB Centre for Primary Care Research, Department of General Practice, Royal College of Surgeons in Ireland, Dublin, Ireland 
${ }^{2}$ HRB Collaboration in Ireland for Clinical Effectiveness Reviews (HRB-CICER), Health Information and Quality Authority, Dublin, Ireland

${ }^{3}$ National Centre for Pharmacoeconomics, St James's University Teaching Hospital, Dublin, Ireland

${ }^{4}$ Department of Pharmacology and Therapeutics, Trinity Centre for Health Sciences, Trinity College Dublin, Dublin, Ireland

${ }^{5}$ National Quality Improvement Team, Health Service Executive, Dublin, Ireland

\section{Twitter Frank Moriarty @FrankMoriarty}

Collaborators The other members of the GPP Study Group are Michael Barry, Fiona Boland, Sarah Clarke, Karen Finnigan, Maria Daly, Catriona Bradley, Paul Gallagher, Edel Murphy, Andrew Murphy, Patrick Byrne, Aisling Croke and Oscar James.

Contributors SMS conceived the study, and all authors contributed to the design of the study. KC coordinated the intervention delivery and data collection. KC, LM and FM analysed the data and all authors were involved in the interpretation of the data. The manuscript was drafted by FM, and all authors were involved in the critical revision and approval of the final manuscript.

Funding This research was supported by the Health Research Board Research Collaborative for Quality and Patient Safety Award.

\section{Competing interests None declared.}

Patient and public involvement Patients and/or the public were not involved in the design, or conduct, or reporting, or dissemination plans of this research.

\section{Patient consent for publication Not required.}

Ethics approval Ethical approval was granted by the Irish College of General Practitioners, and the study protocol has been published previously.

Provenance and peer review Not commissioned; externally peer reviewed.

Data availability statement Data are available in a public, open access repository. Data and analytical code relating to prescribing issues are available from www.doi. org/10.5281/zenodo.3492198.

Open access This is an open access article distributed in accordance with the Creative Commons Attribution Non Commercial (CC BY-NC 4.0) license, which permits others to distribute, remix, adapt, build upon this work non-commercially, and license their derivative works on different terms, provided the original work is properly cited, appropriate credit is given, any changes made indicated, and the use is non-commercial. See: http://creativecommons.org/licenses/by-nc/4.0/.

ORCID iDs

Tom Fahey http://orcid.org/0000-0002-5896-5783

Frank Moriarty http://orcid.org/0000-0001-9838-3625

\section{REFERENCES}

1 van Oostrom SH, Picavet HSJ, de Bruin SR, et al. Multimorbidity of chronic diseases and health care utilization in general practice. BMC Fam Pract 2014;15:61.

2 Sinnott C, Mc Hugh S, Browne J, et al. GPs' perspectives on the management of patients with multimorbidity: systematic review and synthesis of qualitative research. BMJ Open 2013;3:e003610.

3 Spinewine A, Schmader KE, Barber N, et al. Appropriate prescribing in elderly people: how well can it be measured and optimised? Lancet 2007;370:173-84.

4 Sheikh A, Dhingra-Kumar N, Kelley E, et al. The third global patient safety challenge: tackling medication-related harm. Bull World Health Organ 2017;95:546-546A.

5 World Health Organisation. Medication safety in polypharmacy. Geneva: World Health Organization, 2019. http://apps.who.int/ bookorders

6 Clyne B, Fitzgerald C, Quinlan A, et al. Interventions to address potentially inappropriate prescribing in community-dwelling older adults: a systematic review of randomized controlled trials. J Am Geriatr Soc 2016;64:1210-22.

7 Rankin A, Cadogan CA, Patterson SM, et al. Interventions to improve the appropriate use of polypharmacy for older people. Cochrane Database Syst Rev 2018;9:CD008165.

8 Saint-Pierre C, Herskovic V, Sepúlveda M. Multidisciplinary collaboration in primary care: a systematic review. Fam Pract 2018;35:132-41.

9 Nelson P, Martindale A-M, McBride A, et al. Skill-mix change and the general practice workforce challenge. Br J Gen Pract 2018;68:66-7.
10 Hazen ACM, de Bont AA, Boelman L, et al. The degree of integration of non-dispensing pharmacists in primary care practice and the impact on health outcomes: a systematic review. Res Soc Adm Pharm 2018;14:228-40.

11 Tan ECK, Stewart K, Elliott RA, et al. Pharmacist services provided in general practice clinics: a systematic review and meta-analysis. Res Social Adm Pharm 2014;10:608-22.

12 Sloeserwij VM, Hazen ACM, Zwart DLM, et al. Effects of nondispensing pharmacists integrated in general practice on medicationrelated hospitalisations. Br J Clin Pharmacol 2019;85:2321-31.

13 Maskrey M, Johnson CF, Cormack J, et al. Releasing GP capacity with pharmacy prescribing support and new ways of working: a prospective observational cohort study. $\mathrm{Br} J$ Gen Pract 2018;68:e735-42.

14 Bradley F, Ashcroft DM, Noyce PR. Integration and differentiation: a conceptual model of general practitioner and community pharmacist collaboration. Res Soc Adm Pharm 2012;8:36-46.

15 Kwint H-F, Bermingham L, Faber A, et al. The relationship between the extent of collaboration of general practitioners and pharmacists and the implementation of recommendations arising from medication review: a systematic review. Drugs Aging 2013;30:91-102.

16 Deeks LS, Naunton M, Tay GH, et al. What can pharmacists do in general practice? A pilot trial. Aust J Gen Pract 2018;47:545-9 http:// www.ncbi.nlm.nih.gov/pubmed/30114892

17 Cardwell K, Clyne B, Moriarty F, et al. Supporting prescribing in Irish primary care: protocol for a non-randomised pilot study of a general practice pharmacist (Gpp) intervention to optimise prescribing in primary care. Pilot Feasibility Stud 2018;4:122.

18 Eldridge SM, Chan CL, Campbell MJ, et al. Consort 2010 statement: extension to randomised pilot and feasibility trials. BMJ 2016;355:i5239.

19 HSE Medicines Management Programme. Clinical Strategy and Programmes Division - Prescribing and Cost Guidance - Ireland's Health Service.. Available: https://www.hse.ie/eng/about/who/cspd/ ncps/medicines-management/guidance/

20 HSE Medicines Management Programme. Clinical Strategy and Programmes Division - Preferred Drugs - Ireland's Health Service. Available: https://www.hse.ie/eng/about/who/cspd/ncps/medicinesmanagement/preferred-drugs/

21 Clyne B, Smith SM, Hughes CM, et al. Effectiveness of a multifaceted intervention for potentially inappropriate prescribing in older patients in primary care: a cluster-randomized controlled trial (OPTI-SCRIPT study). Ann Fam Med 2015;13:545-53.

22 Guthrie B, McCowan C, Davey $\mathrm{P}$, et al. High risk prescribing in primary care patients particularly vulnerable to adverse drug events: cross sectional population database analysis in Scottish general practice. BMJ 2011;342:d3514.

23 McCarthy C, Clyne B, Corrigan D, et al. Supporting prescribing in older people with multimorbidity and significant polypharmacy in primary care (SPPiRE): a cluster randomised controlled trial protocol and pilot. Implement Sci 2017;12:99.

24 Clyne B, Smith SM, Hughes CM, et al. Effectiveness of a multifaceted intervention for potentially inappropriate prescribing in older patients in primary care: a cluster-randomized controlled trial (OPTI-SCRIPT study). Ann Fam Med 2015;13:545-53.

25 Scott IA, Hilmer SN, Reeve E, et al. Reducing inappropriate polypharmacy. JAMA Intern Med 2015;175:827.

26 Herdman M, Gudex C, Lloyd A, et al. Development and preliminary testing of the new five-level version of EQ-5D (EQ-5D-5L). Qual Life Res 2011;20:1727-36.

27 Duncan P, Murphy M, Man M-S, et al. Development and validation of the multimorbidity treatment burden questionnaire (MTBQ). BMJ Open 2018;8:e019413.

28 Reeve E, Low L-F, Shakib S, et al. Development and validation of the revised patients' attitudes towards deprescribing (rPATD) questionnaire: versions for older adults and caregivers. Drugs Aging 2016;33:913-28.

29 Devlin NJ, Parkin D, Browne J. Patient-Reported outcome measures in the NHS: new methods for analysing and reporting EQ-5D data. Health Econ 2010;19:886-905.

30 Hobbins A, Barry L, Kelleher D, et al. Utility values for health states in Ireland: a value set for the EQ-5D-5L. Pharmacoeconomics 2018;36:1345-53.

31 Health Service Exectutive Primary Care Reimbursement Service. List of Reimbursable Items [Internet]. Available: https://www.sspcrs.ie/ druglist/ [Accessed 20 Sep 2018]

32 National Centre for Pharmacoeconomics. Guidelines for inclusion of drug costs in pharmacoeconomic evaluations version 2.0. Dublin, Ireland: National Centre for Pharmacoeconomics, 2018.

33 Deeks LS, Kosari S, Naunton M. Clinical pharmacists in general practice. Br J Gen Pract 2018;68:320.1-320. 
34 Gillespie RJ, Harrison L, Mullan J. Deprescribing medications for older adults in the primary care context: a mixed studies review. Health Sci Rep 2018;1:e45.

35 Anderson K, Stowasser D, Freeman C, et al. Prescriber barriers and enablers to minimising potentially inappropriate medications in adults: a systematic review and thematic synthesis. BMJ Open 2014;4:e006544.

36 Snell R, Langran T, Donyai P. Patient views about polypharmacy medication review clinics run by clinical pharmacists in GP practices. Int J Clin Pharm 2017;39:1162-5.
37 Buffel du Vaure C, Ravaud P, Baron G, et al. Potential workload in applying clinical practice guidelines for patients with chronic conditions and multimorbidity: a systematic analysis. BMJ Open 2016;6:e010119.

38 Verdoorn S, Kwint H-F, Blom JW, et al. Effects of a clinical medication review focused on personal goals, quality of life, and health problems in older persons with polypharmacy: a randomised controlled trial (DREAMeR-study). PLoS Med 2019;16:e1002798. 